\title{
Nicolau Syndrome after Intramuscular Aminoglycoside Injection
}

\author{
Jun Hyeok Kim (D), Jung Hyeou Kim (D), Seong Ae Kim 미, Seung Eun Baek (D), Deuk-Young Oh (D) \\ Department of Plastic and Reconstructive Surgery, College of Medicine, The Catholic University of Korea, Seoul, Korea
}

\begin{abstract}
Nicolau syndrome is a rare iatrogenic adverse reaction caused by intramuscular injection of various medications. The clinical features include pain, erythematous swelling, infection, and local ischemic necrosis, but the pathogenesis is uncertain. This study presents the first case of Nicolau syndrome that presented with skin and soft tissue necrosis on both buttocks caused by the antibiotic agent kanamycin, which is used to treat multidrug-resistant tuberculosis. A 62-year-old man was referred due to infectious skin and soft tissue necrosis on both buttock areas. He received intramuscular kanamycin injections to both buttocks to treat pulmonary tuberculosis 4 months before presentation. After extensive debridement, skin and soft tissue defects sized approximately $10.0 \times 6.0 \mathrm{~cm}$ and $5.0 \times 5.0 \mathrm{~cm}$ were found on the right and left buttock, respectively, and two transpositional fasciocutaneous flaps were designed to cover the defects. The wounds healed without any complications. Nicolau syndrome has various presentations and, in rare cases, can be fatal. In this case, the patient presented with skin and fat necrosis superimposed with an infectious condition, which required immediate and extensive debridement.
\end{abstract}

Keywords: Nicolau syndrome; Necrosis; Injections; Intramuscular; Anti-bacterial agents

\section{Introduction}

Nicolau syndrome, also referred to as embolia cutis medicamentosa, is a rare iatrogenic complication caused by intramuscular injections of various medications [1-3]. It presents with diverse clinical features, including pain at the injection site, hyperemia, erythematous swelling, superficial infection, and local ischemic necrosis involving both skin and adipose tissue [4]. Although the pathogenesis is uncertain, histologic findings include thrombosed vessels in the reticular dermis [1], and it is hypothesized that the condition is associated with vascular damage or contractions following injection, occlusion by embolism, sympathetic nerve stimulation, and prostaglandin synthesis blocking [2]. Some literature have indicated that various drugs have been associated with Nicolau syndrome including corticosteroids, nonsteroidal anti-inflammatory drugs, recombination interferons, sedatives, iodine sclerosing substances, and local anesthetic agents [3-8].

This study presents the first case of Nicolau syndrome including skin and soft tissue necrosis on both buttocks caused by the antibiotic agent kanamycin. The drug is an aminoglycoside that is currently used as an antibiotic agent for multidrug-resistant tuberculosis. Although common side effects include hearing loss, peripheral neuropathy, and pain at the injection site, this is the first study to associate it with Nicolau syndrome [9]. The present study was approved by the Institutional Review Board (IRB) of our medical institution (IRB No. KC20ZASI0330). The patient provided written informed consent for the publication and the use of his images.

\section{Case Report}

Received: April 10, 2020

Revised: June 12, 2020

Accepted: June 12, 2020

\section{Corresponding author:} Deuk-Young Oh, M.D., Ph.D.

Department of Plastic and Reconstructive Surgery, Seoul St. Mary's Hospital, College of Medicine, The Catholic University of Korea, 222 Banpo-daero, Seocho-gu, Seoul 06591 Korea

Tel: +82-2-2258-6143

Fax: +82-2-594-7230

E-mail: ohdeuk1234@hanmail.net

This is an Open Access article distributed under the terms of the Creative Commons Attribution Non-Commercia License (https://creativecommons.org/licenses/by-nc/4.0/) which permits unrestricted non-commercial use, distribution, and reproduction in any medium, provided the original work is properly cited.

(c) 2020 Korean Wound Management Society 
Kim JH et al.

Nicolau syndrome by aminoglycoside

\section{Case}

A 62-year-old man was referred for infectious skin and soft tissue necrosis on both buttocks. His past medical records showed rheumatoid arthritis and pneumoconiosis, and he had received intramuscular injections of kanamycin, an aminoglycoside, as an alternative to rifampin, to both buttocks to treat pulmonary tuberculosis 4 months prior. A few weeks after the
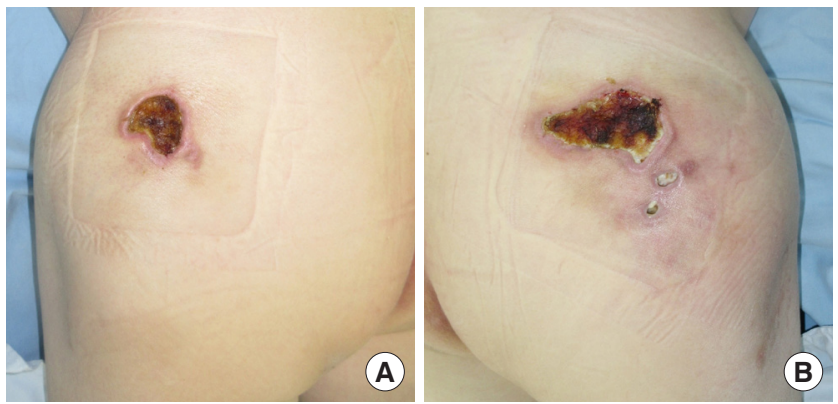

Fig. 1. Clinical photographs before surgery. (A) Left buttock, (B) right buttock.
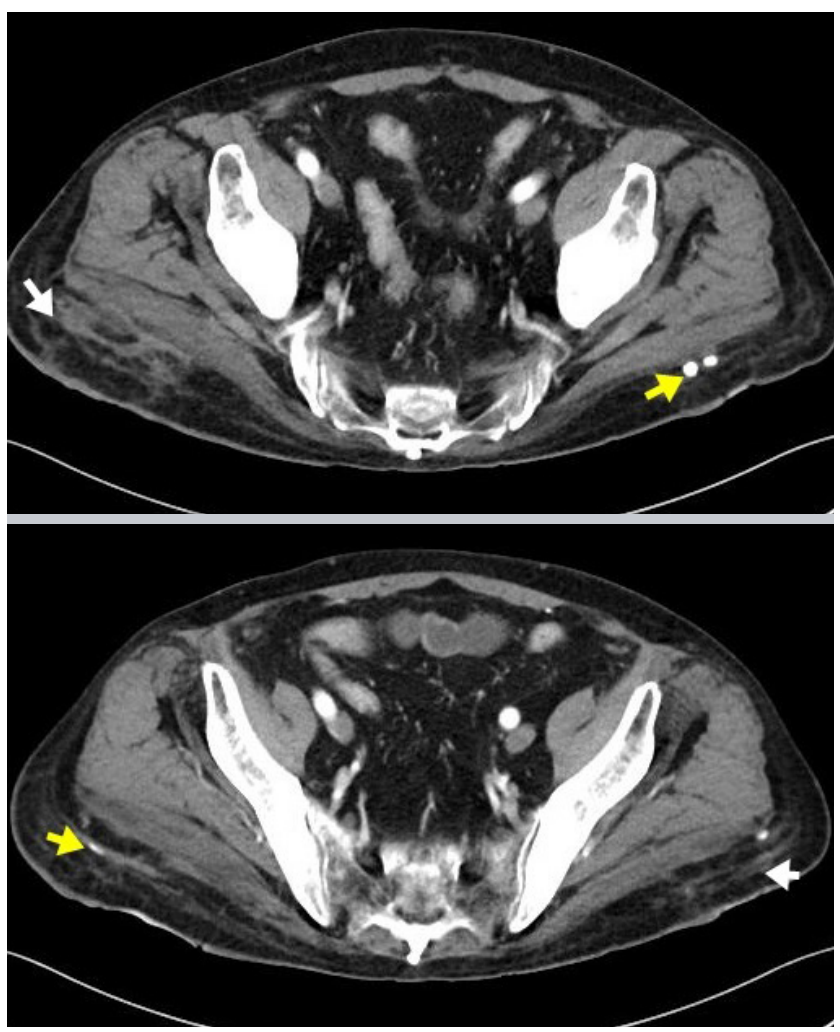

Fig. 2. Preoperative computed tomography. Diffuse inflammation at the adipose tissue layer, focally-enhanced gluteal fascia (indicated by white arrow), and multiple calcified granulomas (indicated by yellow arrow). treatment, the injection sites were necrotized, and the lesions had become larger, but the patient had received only conservative care.

Gross examination showed a $3.0 \times 3.0-\mathrm{cm}$-sized wound on the left buttock and a $6.0 \times 2.5-\mathrm{cm}$-sized wound accompanying two fistulas on the right buttock with skin and adipose tissue necrosis, foul discharge, and marginal erythema with tenderness (Fig. 1). Staphylococcus capitis and Achromobacter xylosoxidans were cultured from the discharge, and a computed tomography scan showed diffuse inflammation at the adipose tissue layer, focally-enhanced gluteal fascia, and multiple calcified granulomas (Fig. 2). Surgery was planned, including extensive debridement of infected tissue and regional fasciocutaneous flap coverage.

Under general anesthesia, the infected tissue was debrided, and diffuse fat necrosis superficial to gluteus muscle was noted, which prompted excision of the necrotized adipose tissue and gluteal fascia. After extensive debridement, skin and soft
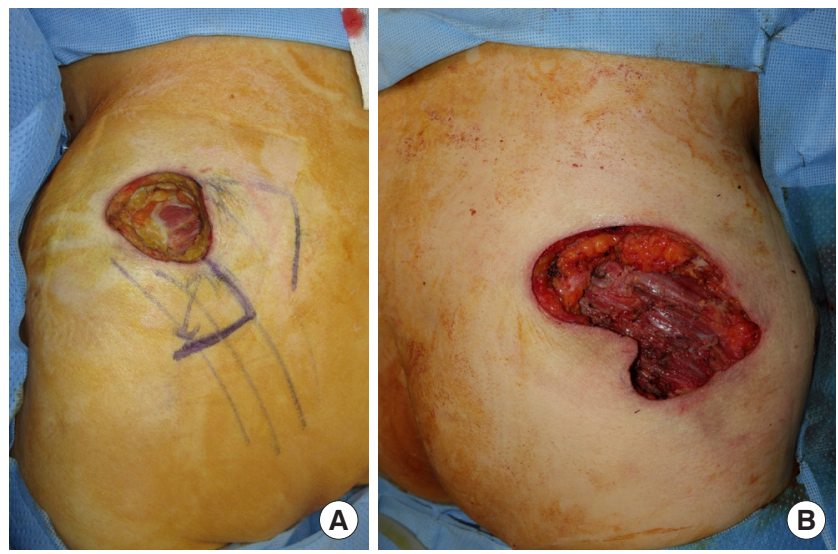

Fig. 3. Intraoperative photographs after extensive debridement. (A) Left buttock, (B) right buttock.
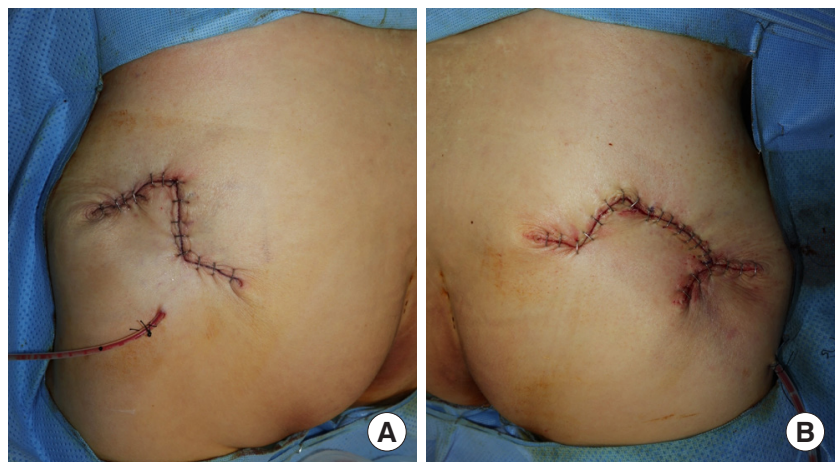

Fig. 4. Immediate postoperative photographs. (A) Left buttock, (B) right buttock. 

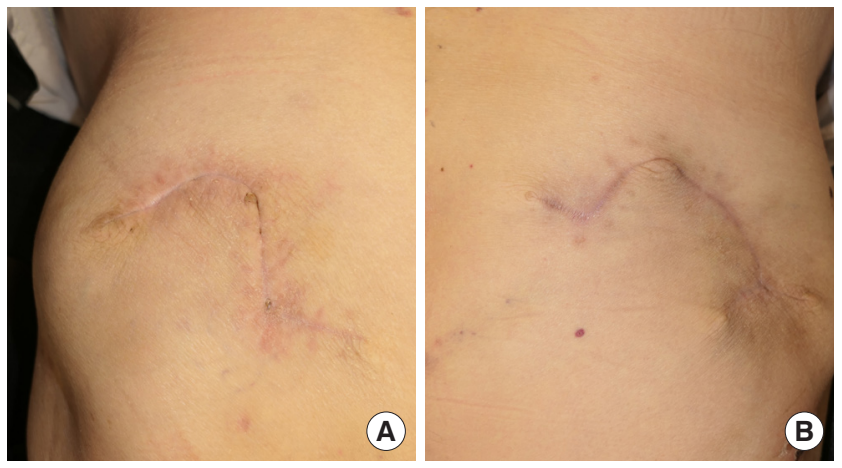

Fig. 5. Three-month postoperative photographs. (A) Left buttock, (B) right buttock.

tissue defects of approximately $10.0 \times 6.0 \mathrm{~cm}$ and $5.0 \times 5.0 \mathrm{~cm}$ were found on the right and left buttock, respectively (Fig. 3). Two transpositional fasciocutaneous flaps were designed to cover both defects, and closed suction drains were inserted on both sides (Fig. 4). After the operation, the patient received intravenous vancomycin and piperacillin-tazobactam for 3 weeks and was discharged from the hospital. The wounds were healed without any complications during three months of follow-up (Fig. 5).

\section{Discussion}

Nicolau syndrome was first described as an adverse reaction to intramuscular injections of bismuth for treating syphilis in the 1920s [4]. It has persisted as a syndrome without an explicitly defined clinical course, and the pathogenesis has not yet been determined. Also, Nicolau syndrome itself is merely an expression of a series of symptoms including pain at the injection site, hyperemia, erythematous swelling, superficial infection, and local ischemic necrosis involving both skin and adipose tissue [4], and the pathogenesis is still uncertain. In addition, in most cases, it is difficult to make an initial intervention because necrosis of tissue is already present when referred to the plastic surgery department for treatment of the wounds. However, Nicolau syndrome is distinguished from common soft tissue inflammation in the injection site, in that it does not have a systemic reaction, such as changes in vital signs or fevers, even in severe cases. In contrast, infection of the injection site to the extent of tissue necrosis can be determined by preceding symptoms such as systemic inflammatory response syndrome.

Nicolau syndrome primarily occurs after intramuscular injection, and it can also be associated with subcutaneous, intra- venous, intra-articular, or subacromial injection [10]. Symptoms associated with its development include tenderness, erythematous swelling, livedo reticularis, and necrosis of skin and soft tissue. Among the intramuscular injection cases, various medications including bismuth [4], vitamin K [6], nonsteroidal anti-inflammatory drugs [4], hydroxyzine [7], vaccination [11], dexamethasone [12], benzathine penicillin [12], penicillin G [1], and cyanocobalamin [1] have been reported as associated drugs. The present case study is the first report of this syndrome caused by intramuscular injection of kanamycin. Based on the clinical course, it is implicit that any type of drug could cause Nicolau syndrome via an intramuscular injection, meaning clinicians should be aware that Nicolaus syndrome can be caused by any drug.

In this patient, rifampicin, isoniazid, ethambutol, and pyrazinamide were used initially to treat pulmonary tuberculosis. Shortly afterwards, as the patient showed nausea, vomiting, and anemic symptoms, rifampicin was excluded and kanamycin was administered. Kanamycin is an aminoglycoside-based antibiotic drug that is not a primary choice for preemptive tuberculosis treatment, but rather an alternative to avoid side effects, and also for multidrug (i.e., both isoniazid and rifampicin) resistant tuberculosis.

The necrotized wounds from Nicolau syndrome vary from erythema to deep tissue necrosis. Therefore, it can be treated conservatively without antibiotic treatment $[10,12]$, and warm compression and massage can also be helpful [2]. However, with deeper necrosis a surgical approach is needed as well, including wide debridement with a negative-pressure wound (NPWT) [4], split-thickness skin graft with or without serial application of NPWT [5,7], direct closure [4,5], or locoregional flaps $[5,13]$. The reported cases indicated that surgery was performed only in severe cases where tissue necrosis was followed by infection.

Although Nicolau syndrome is a rare complication, it is a condition that requires the awareness of clinicians, along with preventive and relieving methods thereof. To reduce the risk of tissue damage, the injection needle should be long enough to reach the intramuscular layer and not leak any drugs in the subcutaneous layer $[5,14,15]$. Local cold compression, which can lead to rapid necrosis, should be avoided where there is immediate, intense pain, and at any erythematous reticular patch arising after intramuscular injection of any drug [14]. Moreover, regurgitation of the syringe only into the muscular layer should be ensured, and the Z-track method of intramuscular injection, which is a traction technique that laterally dis- 
places the skin and subcutaneous layer from the underlying muscle, can be applied to minimize subcutaneous irritation by blocking the needle track after injection $[5,15]$.

\section{Conflict of interest}

No potential conflicts of interest relevant to this article are reported.

\section{ORCID iDs}

Jun Hyeok Kim

Jung Hyeou Kim

Seong Ae Kim

Seung Eun Baek

Deuk-Young Oh https://orcid.org/0000-0003-4657-2090

https://orcid.org/0000-0002-2662-3631

https://orcid.org/0000-0002-4457-8416

https://orcid.org/0000-0003-0873-1636

https://orcid.org/0000-0003-3499-1554

\section{References}

1. Luton K, Garcia C, Poletti E, et al. Nicolau syndrome: three cases and review. Int J Dermatol 2006;45:1326-8.

2. Memarian S, Gharib B, Gharagozlou M, et al. Nicolau syndrome due to penicillin injection: a report of 3 cases without long-term complication. Case Rep Infect Dis 2016;2016 :9082158.

3. Kilic I, Kaya F, Ozdemir AT, et al. Nicolau syndrome due to diclofenac sodium (Voltaren ${ }^{\circledR}$ ) injection: a case report. J Med Case Rep 2014;8:404.

4. Dadaci M, Altuntas Z, Ince B, et al. Nicolau syndrome after intramuscular injection of non-steroidal anti-inflammatory drugs (NSAID). Bosn J Basic Med Sci 2015;15:57-60.

5. Kim SK, Kim TH, Lee KC. Nicolau syndrome after intramuscular injection: 3 cases. Arch Plast Surg 2012;39:249-52.

6. Koklu E, Sarici SU, Altun D, et al. Nicolau syndrome in- duced by intramuscular vitamin $\mathrm{K}$ in a premature newborn. Eur J Pediatr 2009;168:1541-2.

7. Gayken J, Westanmo A, Knutsen A, et al. Livedoid dermatitis and severe necrosis (Nicolau's syndrome) after intramuscular hydroxyzine injection. J Burn Care Res 2006;27: 541-4.

8. Guarneri C, Bevelacqua V, Polimeni G. Embolia cutis medicamentosa (Nicolau syndrome). QJM 2012;105:11278.

9. Momin MAM, Sinha S, Tucker IG, et al. Dry powder formulation of kanamycin with enhanced aerosolization efficiency for drug-resistant tuberculosis. Int J Pharm 2017; 528:107-17.

10. Tabor D, Bertram CG, Williams AJK, et al. Nicolau syndrome (embolia cutis medicamentosa): a rare and poorly recognized iatrogenic cause of cutaneous thrombotic vasculopathy. Am J Dermatopathol 2018;40:212-5.

11. Mueller P, Forner C, Kurze G. Embolia cutis medicamentosa (Nicolau syndrome) due to vaccination in a 2-year-old boy. Klin Padiatr 2012;224:88-9.

12. Marcus F, Claude EV, Josephine M, et al. An exceptional cause of acute limb ischemia: Nicolau syndrome-singlecenter experience with 4 cases. Ann Vasc Surg 2019;58:383.

13. Kocman EA, Yasar FN, Kose AA, et al. Freestyle perforator-based fasciocutaneous flap reconstruction in Nicolau syndrome-related tissue necrosis. Indian J Surg 2015;77: 1187-90.

14. Senel E, Ada S, Gulec AT, et al. Nicolau syndrome aggravated by cold application after i.m. diclofenac. J Dermatol 2008;35:18-20.

15. Lie C, Leung F, Chow SP. Nicolau syndrome following intramuscular diclofenac administration: a case report. J Orthop Surg (Hong Kong) 2006;14:104-7. 